\title{
Condição corporal, variação de peso e desempenho reprodutivo de vacas de cria em pastagem nativa no Pantanal
}

\section{Sandra Aparecida Santos ${ }^{1}$, Urbano Gomes Pinto de Abreu ${ }^{1}$, Geraldo da Silva e Souza ${ }^{2}$, João Batista Catto ${ }^{3}$}

\author{
${ }^{1}$ Embrapa Pantanal, Caixa Postal 109, CEP: 79320-900, Corumbá - MS. \\ 2 Embrapa Sede, Parque Estação Ecológica PqEB s/nº, CEP: 70770-901, Brasília - DF. \\ ${ }^{3}$ Embrapa Gado de Corte, BR 262 km 4 - Caixa Postal 154, CEP: 79002-970, Campo Grande - MS.
}

RESUMO - Objetivou-se avaliar a influência do escore de condição corporal (ECC) sobre a eficiência reprodutiva de vacas de cria nos períodos pré e pós-parto e a probabilidade de parição, usando o escore de condição corporal e a variação ponderal no período crítico do ciclo reprodutivo. Utilizaram-se 248 vacas multíparas mantidas em pastagens nativas distribuídas em quatro estratégias de suplementação: mistura mineral (controle); suplemento farelado; suplemento líquido; suplemento líquido para vacas e bezerros. As avaliações de peso e ECC, na escala de 1 a 9, foram realizadas no período crítico: em agosto (período pré-parto, 60-90 dias antes da parição) e em janeiro (período pós-parto e reprodutivo) durante quatro anos consecutivos. Para a análise dos anos consecutivos de reprodução, criou-se um escore de reprodução, ou seja, somou-se o número de parições de cada vaca durante os quatro anos pecuários avaliados. O escore de condição corporal nos períodos pré e pós-parto e a idade da vaca influenciaram o escore de reprodução. Portanto, para avaliação da probabilidade de parição, foram utilizadas todas as vacas, classificadas em paridas e vazias. Utilizando equações de estimação generalizadas (EEG), foi modelada a probabilidade de as matrizes serem classificadas como paridas considerando variáveis exploratórias ao longo do tempo. Para se obter probabilidade de parição superior a $80 \%$, as vacas devem possuir escore de aproximadamente 5,5 no período pré-parto. Aproximadamente $60 \%$ das vacas paridas mantiveram ou ganharam peso no período crítico do ano, o que levou a maior probabilidade de reconcepção, similar ao observado para as vacas vazias, porém com probabilidade menor de parição. O período pré-parto é o melhor para estimar a probabilidade de parição das vacas por meio da avaliação do escore corporal. A seleção de vacas adaptadas às condições bioclimáticas do Pantanal aumenta a probabilidade de produção de um bezerro por vaca por ano.

Palavras-chave: gado de corte, manejo nutricional, pastagem nativa

\section{Body condition score, weight variation and reproductive performance of beef cows in rangelands from the Pantanal region}

\begin{abstract}
The aim of this study was to evaluate the influence of body condition score (BCS) on reproductive performance of beef cows before and after calving and to evaluate the calving probability using body condition score and weight variation during critical periods of the cow reproductive cycle. From 2001 to 2005, the body condition score was recorded in 248 multiparous cows raised in native pastures, submitted to different supplementation treatments (mineral mixture only - control; dry supplement; liquid supplement and liquid supplement for cow and calves). Weighing and body condition evaluation were made on a 1-9 point scale in August (prepartum period, 60-90 days before parturition) and January (postpartum and reproductive period). A reproduction score was created in function of the body scores in four consecutive years. Except for the treatment, the other factors (body condition score in the prepartum and postpartum periods and cow age) influenced the reproduction score. Thus, the calving probabilities were estimated for all cows. Generalized estimating equations (GEE) were used to evaluate the calving probability in function of the exploratory variables over time. Calving probability higher than $80 \%$ was obtained when cows scored 5,5 before calving. In relation to weight, the results showed that about $60 \%$ of calving cows maintained or gained weight in the postpartum period, leading to increase in the rebreeding probability. Similar situation occurred with single cows; however, the calving probability was lower. These results indicate that the calving probability using BCS is better estimated before calving. Furthermore, it indicates the presence of cows more adapted to the Pantanal climatic and feeding conditions than others.
\end{abstract}

Key Words: beef cattle, natural pastures, nutritional management

Este artigo foi recebido em 18/6/2007 e aprovado em 31/7/2008.

Correspondências devem ser enviadas para: sasantos@cpap.embrapa.br 


\section{Introdução}

O desempenho reprodutivo de vacas de cria na planície pantaneira é fundamental para o aumento da produtividade da bovinocultura de corte, pois bezerros desmamados são um dos principais produtos comercializados pelos produtores rurais da região. Vários autores (Almeida et al., 1996; Afonso et al., 2001; Santos, 2001) têm mostrado que, nos rebanhos de cria do Pantanal, existem vacas que parem todos os anos e vacas que nunca parem ou que parem ocasionalmente, resultando em baixa taxa de fertilidade (em torno de 55\%). Esse índice tem aumentado na região com a adoção de práticas adequadas de manejo e com a seleção de animais adaptados (Abreu et al., 2001).

O desempenho reprodutivo das vacas de cria é influenciado por diversos fatores, como o nutricional, principalmente o balanço de energia, que reflete na reserva de gordura corporal. No período de escassez alimentar, para sustentar o crescimento fetal e a produção de leite, as vacas prenhes ou em lactação necessitam mobilizar reservas corporais de energia e proteína que foram armazenadas na época de abundância de alimentos (Demment \& Van Soest, 1985). Segundo Wright et al. (1998), o grau de mobilização das reservas corporais também depende do genótipo do animal.

No Pantanal da sub-região de Nhecolândia, o principal período de restrição alimentar é de maio a setembro. Visando associar a produtividade das pastagens às necessidades nutricionais das vacas de cria, a estação de monta nesta sub-região foi implantada no período das águas, de outubro a dezembro (Almeida et al.,1996), o que possibilita a concentração de nascimentos dos bezerros no início do período das águas. Portanto, a taxa de prenhez é estabelecida no período de lactação e a desmama coincide com o início do período de restrição alimentar.

O escore de condição corporal (ECC) é uma medida visual, prática e de baixo custo, usada para monitorar o estado nutricional e o desempenho reprodutivo de matrizes em várias regiões do mundo, pois é determinante na reconcepção e na produtividade geral do rebanho (Richard et al., 1986; Selk et al., 1988). Um programa de manejo nutricional adequado deve proporcionar condição corporal desejável nos diferentes estágios de produção, cujas exigências aumentam significativamente no terço final da gestação e no intervalo de parição (início da lactação) até a reconcepção (pico da lactação). A avaliação do escore de condição corporal permite a análise das práticas de manejo adotadas e pode fornecer subsídios aos produtores na melhoria e na eficiência dos programas de manejo reprodutivo e nutricional.
Objetivou-se com este estudo avaliar a influência do escore de condição corporal sobre a eficiência reprodutiva de vacas de cria nos períodos pré-parto e pós-parto e analisar a probabilidade de parição de vacas nesses períodos, usando o escore de condição corporal e a variação ponderal no período pós-parto, na sub-região de Nhecolândia, Pantanal.

\section{Material e Métodos}

O estudo foi desenvolvido na fazenda Nhumirim, sub-região da Nhecolândia, Pantanal, Mato Grosso do Sul, no período de 2000 a 2005. A fazenda possui unidades de paisagem características da sub-região, como cerradão, campo-cerrado, campo limpo, lagoas e vazantes, dispostas em mosaico. Dessas unidades de paisagem, os bovinos forrageiam principalmente as pastagens localizadas nas áreas mais baixas do mesorelevo, como campo limpo e áreas baixas (bordas de baías e vazantes), cuja disponibilidade e qualidade da pastagem variam conforme a precipitação e o nível de inundação pluvial. No período de estudo, a precipitação anual esteve abaixo da normal climatológica da região, 1.180,8 mm (1977 a 2003); 675,6 mm (2002); 991,8 mm (2003); e 769,8 mm (2004), de acordo com Soriano \& Alves (2005), o que possibilitou maior disponibilidade das pastagens localizadas nas áreas mais baixas e inundáveis, geralmente de melhor qualidade.

Em 2000, 248 matrizes Nelores foram separadas em blocos, de acordo com a idade, o período de gestação e a condição corporal, e distribuídas em quatro invernadas com pastagem nativa, em lotação contínua e leve (0,23 animal/ha). Cada lote, composto de 62 vacas, foi mantido com uma das formas de suplementação: mistura mineral o ano todo (controle); suplemento farelado; suplemento líquido; suplemento líquido às vacas e aos bezerros (uso de cocho privativo). A mistura mineral foi composta de: $\mathrm{P}$ - 87,647 g/kg; $\mathrm{Ca}$ - 121,732 g/kg; NaCl - 108,108 g/kg; Mg - 47,311 g/kg; S - 38,551 g/kg; Zn - 3746,5 mg/kg; Cu - 812,5 mg/kg; Co 16 mg/kg; I - 36 mg/kg; e Se - 15,5 mg/kg. O suplemento farelado era composto de: milho, 20\%; farelo de soja, $37,5 \%$; uréia, $13,4 \%$; sulfato de amônia, 2,6\% e mistura mineral, 26,5\%. O suplemento líquido era composto de: proteína bruta, 25\%; NNP (nitrogênio não-protéico), 3,6\%; NDT (nutrientes digestíveis totais), 50\%; macrominerais, 28,6 g/kg; microminerais, 938 mg/kg; vitaminas ADE, 273.127 UI e outras vitaminas, $200 \mathrm{mg} / \mathrm{kg}$. A quantidade diária de suplemento para as matrizes foi de aproximadamente $400 \mathrm{~g} / \mathrm{dia} / \mathrm{animal}$ para atender ao consumo de aproximadamente três dias, quando era feito o reabastecimento. 
No período de 2000 a 2001, a suplementação foi feita de setembro a dezembro (período pós-parto) e, no período de 2002 a 2005, de maio a setembro (período pré-parto). Para reduzir o efeito de pasto, os lotes foram rotacionados entre as invernadas aproximadamente a cada 28 dias, quando era mensurado o consumo da mistura mineral. Nos meses iniciais da estação de parição, evitou-se o rodízio dos lotes entre as invernadas. Os rebanhos seguiram o manejo sanitário e reprodutivo adotado na fazenda, com estação de monta de dezembro a março, na proporção touro:vaca de 1:25 (Sereno et al., 1996).

As vacas foram acompanhadas durante quatro anos pecuários consecutivos: 2001/2002; 2002/2003; 2003/2004 e 2004/2005 no período pré-parto (agosto), correspondente ao final da estação da seca e 60-90 dias antes do pico da parição, e no período pós-parto (janeiro), correspondente ao período reprodutivo e ao pico da lactação. Nesses períodos, foram feitas avaliações dos escores corporais das vacas, em escala de 1 a 9 (Nicholson \& Butterworth, 1986), por dois observadores devidamente treinados. A perda e o ganho de peso foram avaliados durante os quatros anos, no período de agosto a janeiro do ano subseqüente, que correspondeu ao principal período crítico do ciclo reprodutivo. Para este período, estimou-se a variação diária de peso, usando a seguinte classificação: ganho ou perda diária alta (acima de 500 g); ganho ou perda diária moderada (101 a 500 g); manutenção do peso; perda ou ganho diário baixa (abaixo de $100 \mathrm{~g}$ ).

Para avaliar a influência do ECC sobre a eficiência reprodutiva das vacas de cria no período pré-parto e pósparto, criou-se um escore de reprodução, somando o número de parições de cada vaca durante os quatro anos pecuários consecutivos avaliados. Como foram considerados quatro anos, o escore variou de 1 a 4 . Foram também calculadas as médias dos escores de condição corporal de agosto (período pré-parto) e de janeiro (período pós-parto). Posteriormente, analisou-se o escore de reprodução de acordo com a média dos escores corporais no período pré-parto (ESCPRE), do escore no período pós-parto (ESCPOS), da idade inicial e das formas de suplementação, por meio de uma análise de co-variância não-paramétrica, usando o PROC GLM e a ordenação (ranks) dos escores como resposta. O modelo utilizado foi o seguinte:

Rank $(E R)=a+b * E S C P R E+c * E S C P O S+d * i d a d e+$ $\mathrm{e} * \operatorname{trat} \mathrm{A}+\mathrm{f} * \operatorname{tratB}+\mathrm{g} * \operatorname{trat} \mathrm{C}+\mathrm{h} * \operatorname{trat} \mathrm{D}+$ erro

em que: $\mathrm{ER}$ = escore de reprodução; ESCPRE = ECC préparto; $\mathrm{ESCPOS}=$ ECC pós-parto; idade = idade da vaca no início do estudo; $\mathrm{A}=$ mistura mineral o ano todo; $\mathrm{B}=$ suplementação farelada; C = suplementação líquida; D = suplementação líquida às vacas e aos bezerros.

A probabilidade de uma vaca reproduzir foi então modelada por meio de equações de estimação generalizadas (EGG), que considera as correlações entre as variáveisrespostas em análises nas quais são: dados binários, dados de contagens e dados ordinais (Liang \& Zeger, 1986). Modelou-se a probabilidade de parição de vacas multíparas, por meio do ECC avaliados no período pré e pós-parto e por meio da variação ponderal no período crítico, de agosto a janeiro do ano subseqüente. Como a resposta reprodutiva é de natureza binária, as matrizes foram classificadas como 0 e 1 (vazias e paridas, respectivamente) ao final da estação de reprodução na região. As avaliações dos ECC e de pesos ocorreram no mesmo animal durante os quatro anos pecuários. Conseqüentemente, as variáveis-resposta possuíam correlação intra-animal de natureza binária, de modo que, entre os animais, não havia correlação. Caracterizando a análise longitudinal com repostas binárias repetidas ao longo do tempo, houve a necessidade de modelar a estrutura de co-variância entre as respostas de cada indivíduo analisado e a correlação entre os resíduos, pois, do contrário, aumentaria o risco de realizar inferências científicas incorretas (Diggle et al., 2002). Neste estudo, como as estratégias de suplementação não tiveram efeito sobre o escore de reprodução, o efeito suplementação foi retirado do modelo de análise probabilística. Assim, foram consideradas todas as vacas na análise, independentemente da forma de suplementação. A análise foi realizada utilizando-se o PROC GENMOD do pacote estatístico SAS, com o comando REPEATED, visando modelar as estruturas co-variância intra-indivíduo de acordo com metodologia descrita por Stokes et al. (2000). O modelo estatístico utilizado foi:

$$
\begin{gathered}
\ln \left[\mathrm{P}_{\mathrm{i}} /\left(1-\mathrm{P}_{\mathrm{i}}\right)\right]=\beta_{0}+\beta 1(\text { ECCPreP })+\beta_{2}(\text { ECCPosP })+ \\
\beta_{3}(\text { ID }) ;
\end{gathered}
$$

em que $\mathrm{i}$ = i-ésima matriz; $\mathrm{P}=$ probabilidade de a vaca ser classificada como parida (1); $\beta_{0}=$ termo de intercepto; e $\beta_{1}$, $\beta_{2}$, e $\beta_{3}=$ coeficientes estimados por meio de EEG, associados a cada variável exploratória. ECCPreP e ECCPosP = escores de condição corporal pré-parto e pósparto, respectivamente; ID = idade estimada, em anos, da matriz durante o desenvolvimento do experimento.

Para verificar o valor em quilos da mudança de uma unidade de escore de condição corporal, foram ajustadas equações de regressão para estimativa de peso e erropadrão, cuja variável-dependente foi a diferença, em quilos, e a variável independente, a diferença de escore, no período crítico reprodutivo, de agosto a janeiro do ano subseqüente. 


\section{Resultados e Discussão}

O escore de condição corporal nos períodos pré e pósparto e a idade da vaca influenciaram o escore de reprodução, ou seja, o número de parições durante os quatro anos consecutivos avaliados (Tabela 1). Como o efeito da estratégia de suplementação não teve influência sobre a eficiência reprodutiva, provavelmente a condição corporal foi influenciada principalmente pela qualidade e disponibilidade das pastagens nativas, que foram variáveis entre anos. Estudo realizado por Santos et al. (2002) comprovaram que as vacas de cria mantidas em pastagem nativa no Pantanal apresentaram melhor desempenho quando a dieta continha cerca de $10 \%$ de proteína bruta e digestibilidade in vitro acima de $60 \%$. As forrageiras com esta qualidade e em quantidades apropriadas foram suficientes para manter as vacas em condição corporal adequada (acima de 5,0) no período pré-parto.

Para obter probabilidade acima de $80 \%$ de parição, as vacas devem possuir escore aproximado de 5,5 no período pré-parto e 6,0 no período pós-parto (Figura 1). As probabilidades de as vacas estarem prenhes quando ECC foi avaliada em agosto (pré-parto) apresentaram tendência quadrática. As probabilidades aumentam conforme o valor dos ECC e chegam a mais de $90 \%$ quando ECC ultrapassa o escore 6,0. A partir do escore 7,0, a probabilidade é máxima e se estabiliza em ambos os períodos, o que não justifica ter vacas com escore acima desse valor. As taxas de parição foram de 65,0; 54,0; 66,0 e 75,0 \%, respectivamente, para os anos pecuários de 2001/2002, 2002/2003, 2003/2004 e 2004/2005 (Tabela 2).

Na sub-região de Nhecolândia, o período crítico de restrição alimentar compreende os meses mais secos do ano, entre maio e setembro (Santos et al., 2002), cujo pico da seca ocorre em agosto/setembro, que corresponde ao período crítico pré-parto, 60-90 dias antes da estação da parição, que neste estudo concentrou-se nos meses de outubro-novembro. Portanto, os resultados obtidos indicam que a avaliação do ECC neste período é um

Tabela 1 - Análise da variância do escore de reprodução ${ }^{1}$ de vacas de cria nos quatro anos pecuários, de 2001 a 2005, na sub-região da Nhecolândia, Pantanal

\begin{tabular}{lcc}
\hline Fonte de variação & Grau de liberdade & Quadrado médio \\
\hline Escore pré-parto & 1 & $5.832^{* *}$ \\
Escore pós-parto & 1 & $8.958^{* *}$ \\
Idade da vaca & 1 & $2.609^{*}$ \\
Suplementação & 3 & $0.808^{\mathrm{NS}}$ \\
\hline
\end{tabular}

${ }^{1}$ Escore de reprodução = número de parições $(1$ a 4).

** Significativo a $1 \%(\mathrm{P}<0,01)$; * Significativo a $5 \%(\mathrm{P}<0,05)$; NS: não-significativo. interessante indicador de que a matriz irá parir ou não na estação de nascimento subseqüente, o que permite ao produtor implementar estratégias de manejo adequadas para melhorar a eficiência reprodutiva. Esses resultados estão de acordo com outros estudos, como os de Randel (1990) e Santos et al. (2002). Os valores médios de mudanças de uma unidade de ECC das vacas Nelore nos diferentes anos pecuários estão dentro da faixa de 30 a $40 \mathrm{~kg}$, citada por Swecker (1997) para raças de corte européia, na escala de 1 a 9 (Tabela 2).

A característica produtiva mais sensível de uma vaca em reprodução é a taxa de reconcepção, pois é a última a receber energia disponível, que segue a seguinte ordem de importância: metabolismo basal, atividade, crescimento, reservas de energia, prenhez, lactação, reservas adicionais de energia, ciclo estral e início de prenhez e reservas de energia em excesso (Short \& Adams, 1988). Como no período pré-parto (60-90 dias antes da parição) as exigências energéticas das vacas de corte ainda são baixas, esse é o período mais adequado para adotar estratégias de manejo nutricional, pois nesta fase as vacas podem armazenar gordura de forma mais rápida e econômica. Neste período, as matrizes devem ser separadas por meio do ECC, ou seja, as vacas com ECC abaixo de 5,0-5,5 devem ser colocadas em pastagens de melhor qualidade e/ou receber um plano de manejo nutricional adequado. Conforme descrito por Boadi \& Price (1996), as exigências dietéticas antes da parição são difíceis de determinar, uma vez que as respostas das vacas diferem conforme o ECC, o tamanho, a idade e o potencial de produção de leite. Por outro lado, a época mais adequada para realizar a venda de vacas gordas e vazias no Pantanal é em janeiro, o que torna importante a realização de diagnós-

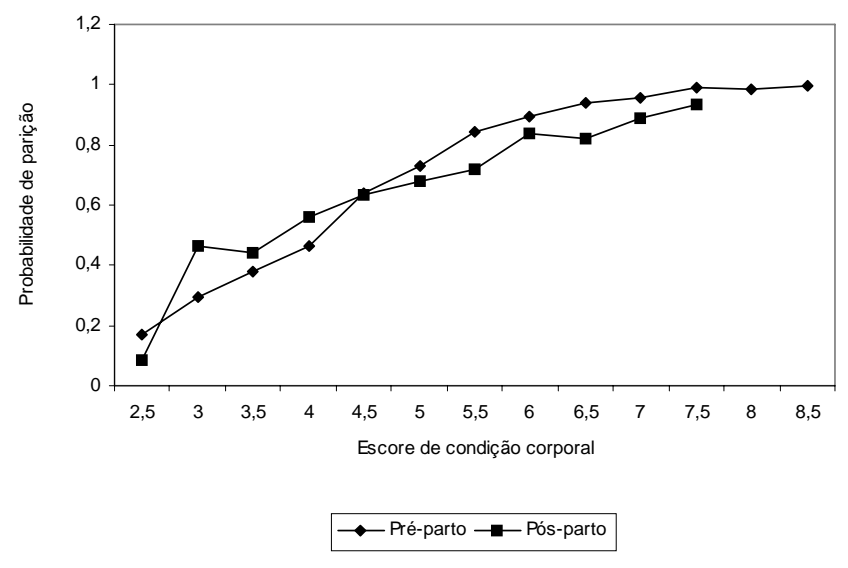

Figura 1 - Probabilidade de parição de vacas multíparas no período pré e pós-parto, de acordo com o escore de condição corporal, no período de 2001 a 2005, na sub-região da Nhecolândia, Pantanal. 
Tabela 2 - Taxa de parição, valores médios e respectivos desvios-padrão de escore de condição corporal, peso corporal e valor unitário de escore de vacas multíparas, no período pecuário de 2001 a 2005, sub-região da Nhecolândia, Pantanal

\begin{tabular}{lcccc}
\hline Parâmetro/Período & $2001 / 2002$ & $2002 / 2003$ & $2003 / 2004$ & $2004 / 2005$ \\
\hline Taxa de parição (\%) & 65,0 & 54,0 & 66,0 & 75,0 \\
Escore pré-parto (1-9) & $4,3 \pm 1,0$ & $4,5 \pm 1,0$ & $5,1 \pm 1,0$ & $5,7 \pm 1,0$ \\
Peso pré-parto (kg) & $377,0 \pm 45,0$ & $374,0 \pm 45,0$ & $400,0 \pm 42,0$ & $420,0 \pm 41,0$ \\
Escore pós-parto (1-9) & $4,9 \pm 1,0$ & $5,1 \pm 1,0$ & $5,4 \pm 1,0$ & $5,4 \pm 1,0$ \\
Peso pós-parto (kg) & $394,0 \pm 38,0$ & $395,0 \pm 38,0$ & $410,0 \pm 37,0$ & $408,0 \pm 38,0$ \\
Valor unitário de escore (kg) ${ }^{1}$ & $35,2 \pm 1,3$ & $38,5 \pm 1,2$ & $32,5 \pm 0,8$ & $35,2 \pm 1,1$ \\
\hline
\end{tabular}

${ }^{1}$ Valor de mudança de uma unidade de escore de condição corporal no período de agosto a janeiro do ano subseqüente.

tico de gestação para certificar que as fêmeas realmente estão vazias, pois, apesar de menor, a probabilidade de a vaca estar prenhe existe.

O mês de janeiro corresponde ao pico do período chuvoso, quando geralmente as pastagens apresentam maior disponibilidade de matéria seca. Na avaliação do ECC em janeiro (período pós-parto), foram observadas as maiores variações na resposta, por exemplo, matrizes com ECC 3,0 tiveram maior probabilidade de parir que matrizes com ECC 3,5. Entretanto, observou-se que, para as vacas com o ECC a partir de 4,5, a probabilidade de prenhez do ECC avaliado em agosto é maior que do ECC avaliado em janeiro. Esse fato pode estar relacionado a um confundimento entre as vacas gestantes e vazias que apresentam ECC acima de 4,5, principalmente no período de janeiro, quando as pastagens são de maior disponibilidade e qualidade. As vacas vazias podem ser consideradas subférteis e que regularmente falham no período reprodutivo, não produzindo um bezerro por ano e contribuindo para o aumento do intervalo médio de partos do rebanho.

Embora as variações no peso e na condição corporal possam ser manipuladas mais eficientemente durante a prenhez (período pré-parto) que após o parto, estratégias de manejo adequadas devem ser adotadas em ambos os períodos, pois há interação conhecida da nutrição pré e pós-parto (Rosa, 1993), devendo-se evitar perdas de peso vivo severo entre o período da parição e o início da reprodução. Cantrell et al. (1981) observaram que a perda de peso após a parição (acima de 10\%) diminuiu a taxa de concepção (taxa de fertilidade).

Considerando que um dos principais objetivos do produtor é que a vaca produza um bezerro por ano, os dados deste estudo mostram que a vaca parida tem maior probabilidade de reconcepção quando apresenta ganho ou manutenção do peso no período critico da reprodução. Lalman et al. (1997) verificaram que novilhas de corte de primeira cria que estavam ganhando 1,8 unidades de ECC durante os primeiros 90 dias de lactação tiveram redução no intervalo pós-parição e atividade lútea.

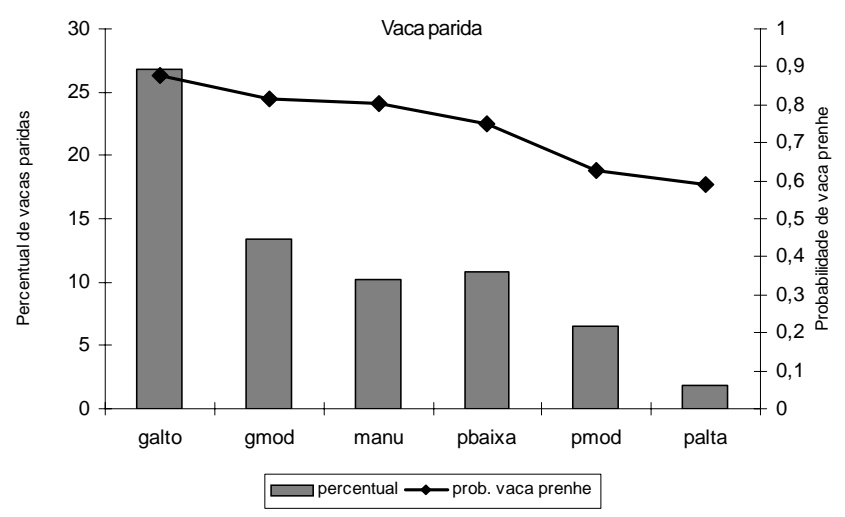

Figura 2 - Porcentagem de vacas paridas e respectiva probabilidade de parir, de acordo com a classe de variação de peso, na sub-região de Nhecolândia, Pantanal, no período de agosto a fevereiro nos anos de 2001 a 2005 (galto = ganho diário acima de 500 g; gmod $=$ ganho diário de 101 a $500 \mathrm{~g}$; manu = manutenção do peso; pbaixa = perda diária abaixo de 100 g; pmod = perda diária de 101 a 500 g; palta = perda diária acima de 500 g).

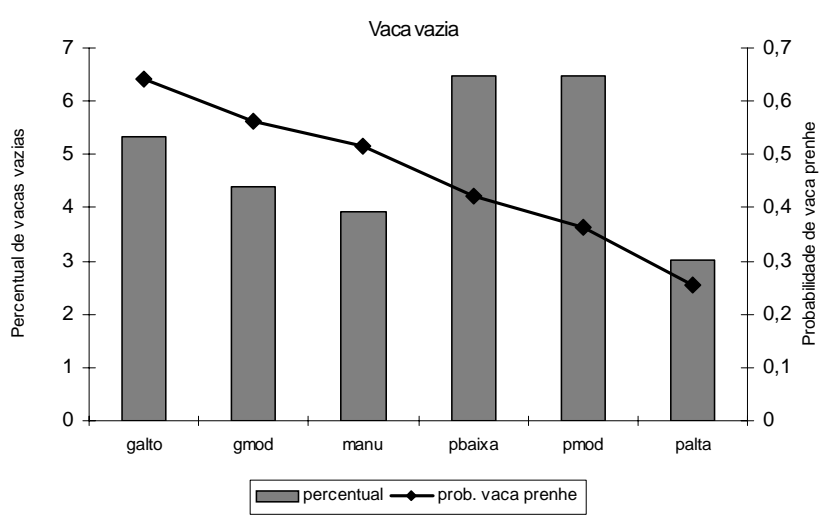

Figura 3 - Porcentagem de vacas vazias e a respectiva probabilidade de parir, de acordo com a classe de variação de peso, na sub-região de Nhecolândia, Pantanal, no período de agosto a fevereiro de 2001 a 2005. (galto = ganho diário acima de 500 g; gmod = ganho diário de 101 a 500 g; manutenção do peso; pbaixa = perda diária abaixo de 100 g; pmod = perda diária de 101 a 500g; palta = perda diária acima de 500 g). 
Neste estudo, as vacas paridas com altas perdas de peso (acima de $500 \mathrm{~g} / \mathrm{dia}$ ) apresentaram probabilidade muito baixa de reconcepção (abaixo de 10\%). Segundo Cantrell et al. (1981), vacas que continuam a perder peso após a parição geralmente apresentam atraso de 80 a 100 dias na manifestação do primeiro cio, resultando em taxas de prenhez 30-50\% mais baixas.

A probabilidade de parição das vacas vazias também foi influenciada significativamente $(\mathrm{P}<0,01)$ pelo ganho de peso, porém, a probabilidade de as matrizes vazias parirem mesmo ganhando peso é mais baixa que a das vacas paridas, o que indica diferenças de potencial produtivo entre vacas e a possibilidade de manter no rebanho de cria as vacas mais adaptadas às condições ambientais para permitir ganho de peso em pastagens nativas.

Houve um percentual de vacas vazias que, mesmo ganhando peso, não ficaram gestantes e, apesar de apresentarem probabilidade de parirem na estação de nascimento subseqüente, não pariram. Portanto, este é o percentual de animais que podem ser descartados, pois provavelmente estarão em boa condição corporal ao final da estação reprodutiva.

As vacas de cria no Pantanal devem ganhar peso no período crítico da reprodução para aumentar a probabilidade de parição. Portanto, as vacas devem ser monitoradas neste período para assegurar balanço nutricional positivo. A seleção das vacas deve ser realizada por meio da manutenção das matrizes mais adaptadas aos recursos forrageiros nativos e às condições climáticas da região, ou seja, para que consigam manter ou ganhar peso no período pós-parto. Paralelamente, estudos complementares devem ser feitos para verificar a probabilidade de parição considerando o tamanho das vacas.

Conforme descrito por Santos (2001), a decisão de quando, onde e como fornecer suplemento às vacas de cria no Pantanal não deveria ser pré-fixada, pois o consumo está relacionado à quantidade e qualidade do pasto nativo, que varia não somente entre locais, mas entre anos, em razão da intensidade e duração da precipitação, o que torna necessário estabelecer estratégias adaptativas de manejo nutricional e das pastagens, de modo que as reais necessidades nutritivas sejam supridas nas diferentes fases de produção.

\section{Conclusões}

A avaliação do escore de condição corporal nos períodos críticos do ciclo reprodutivo mostrou-se eficaz e prática para ser implementada como rotina no manejo do rebanho de cria do Pantanal. A avaliação do escore de condição corporal pré-parto (60-90 dias antes do período de parição) é um bom indicador de parição na estação de nascimento subseqüente. Por outro lado, a avaliação do escore de condição corporal pós-parto (pico da lactação e período reprodutivo), em conjunto com o diagnóstico de gestação, é a prática adequada para a realização de venda de vacas gordas (descarte) no Pantanal. A definição de um plano de manejo nutricional estratégico no período pré e pós-parto, associada à seleção de vacas adaptadas às condições bioclimáticas do Pantanal, aumenta a probabilidade de as vacas produzirem um bezerro por ano.

\section{Agradecimentos}

À FUNDECT, PRODETAB, EMBRAPA, CPP e Anipro do Brasil, pelo financiamento deste trabalho; ao Dr. Luiz Roberto Lopes de S. Thiago e ao Dr. José Marques da Silva, pesquisadores da Embrapa Gado de Corte; ao pesquisador Dr. Aníbal Comastri Filho e aos técnicos e operários rurais da fazenda Nhumirim, Embrapa Pantanal.

\section{Literatura Citada}

ABREU, U.G.P.; MORAES, A.S.; SEIDL, A.F. Tecnologias apropriadas para o desenvolvimento da bovinocultura de corte no Pantanal. Corumbá: Embrapa Pantanal, 2001. 31p. (Documentos, 24).

ALMEIDA, I.L.; ABREU, U.G.P.; LOUREIRO, J.M.F. et al. Introdução de tecnologias na criação de bovino de corte no Pantanal - sub-região dos Paiaguás. Corumbá: EMBRAPA-CPAP, 1996. 50p. (Circular Técnica, 22).

AFONSO, E.; CATTO, J.B.; POTT, E.B. et al. Suplementação mineral para vacas de cria no Pantanal Mato-Grossense. Corumbá: Embrapa Pantanal, 2001. 6p. (Comunicado Técnico, 25).

BOADI, D.; PRICE, M.A. The effects of pre- and post-calving management on reproductive performance of beef cows. Canadian Journal of Animal Science, v.76, p.337-342, 1996.

CANTRELL, J.A.; KROPP, J.R.; ARMBRUSTER, S.L. et al. The influence of postpartum nutrition and weaning age of calves on cow body condition, estrus, conception rate and calf performance of fall-calving beef cows. Oklahoma Agricultural Experimental Station. MP 112, 1981, p.53-58.

DEMMENT, M.W.; Van SOEST, P.J. A nutritional explanation for body-size patterns of ruminant and nonruminant herbivores. The American Naturalist, v.125, n.5, p.641-672, 1985.

DigGle, P.J.; HeAGERTY, P.; LIANG, K.Y. et al. Analysis of longitudinal data. Oxford: Oxford University Press, 2002. 379p.

LALMAN, D.L.; KEISLER, D.H.; WILLIANS, J.E. et al. Influence of pospartum weight and body condition change on duration of anestrus by undernourished suckled beef heifers. Journal of Animal Science, v.75, p.1715-1722, 1997.

LIANG, K.Y.; ZEGER, S.L. Longitudinal data analysis using generalized linear models. Biometrika, n.73, p.13-22, 1986.

NICHOLSON, M.J.; BUTTERWORTH, M.H. A guide to condition scoring of Zebu cattle. Addis Ababa: International Livestock Centre for Africa, 1986. 29p.

RANDEL, R.D. Nutrition and postpartum rebreeding in cattle. Journal of Animal Science, v.68, n.3, p.853-862, 1990. 
RICHARD, M.W.; SPITZER, J.C.; WARNER, M.B. Effect of varying levels of pospartum nutrition and body condition at calving on subsequent reproductive performance in beef cattle. Journal of Animal Science, v.62, n.2, p.300-306, 1986.

ROSA, I.V. Deficiências minerais e desempenho reprodutivo de ruminantes. Campo Grande: Embrapa Gado de Corte, 1993. (Circular Técnica, 23).

SANTOS, S.A. Caracterização dos recursos forrageiros nativos da sub-região da Nhecolândia, Pantanal, Mato Grosso do Sul, Brasil. 2001. 190f. Tese (Doutorado em Nutrição e Produção Animal) - Universidade Estadual Paulista, Botucatu, 2001.

SANTOS, S.A.; COSTA, C.; SOUZA, G.S.E. et al. Qualidade da dieta selecionada por bovinos na sub-região da Nhecolândia, Pantanal. Revista Brasileira de Zootecnia, v.31, n.4, p.663-1673, 2002

SELK, G.E.; WETTEMANN, R.P.; LUSBY, K.S. et al. Relationships among weight change, body condition and reproductive performance of range beef cows. Journal of Animal Science, v.66, p.3153-3159, 1988

SERENO, J.R.B.; PORTO, J.C.A.; CURVO, J.B.E. et al. Efeito da duração do período de monta sobre a eficiência reprodutiva de fêmeas da raça nelore no estado de Mato Grosso do Sul. Revista da Sociedade Brasileira de Zootecnia, v.25, p.661-670, 1996.

SORIANO, B.M.A.; ALVES, M.J.M. Boletim agrometeorológico ano 2002 para a sub-região da Nhecolândia, Pantanal, Mato Grosso do Sul, Brasil. Corumbá: Embrapa Pantanal, 2005. 28p. (Documentos, 76).

SHORT, R.E.; ADAMS, D.C. Nutritional and hormonal interrelationhips in beef cattle reproduction. Canadian Journal of Animal Science, v.68, p.29-39, 1988.

STOKES, M.E.; DAVIS, C.S.; KOCH, G.G. Categorical data analysis using SAS system. 2.ed. Cary: 2000. 626p.

SWECKER, W.S. Effects of nutrition on reproductive performance of beef cattle. In: YOUNGQUIST, R.S. (Ed.) Current therapy in large animal theriogenology. Philadelphia: W. B. Saunders Company, 1997. p.423-428.

WRIGHT, I.A. Identifying biological constraints acting on livestock systems in marginal areas. In: LIVESTOCK SYSTEMS IN EUROPEAN RURAL DEVELOPMENT, 1., 1997, Nafplio. Proceedings... Nafplio: LSIRD, 1998. p.11-18. 